\title{
Correlates of problematic internet use among undergraduate medical students of Delhi
}

\author{
Deepak Dhamnetiya*, Satyavir Singh and Ravi Prakash Jha
}

\begin{abstract}
Background: Globally, due to population diversity, the prevalence of problematic internet use (PIU) varies from 7.3 to $51 \%$. This study aims to assess correlates of problematic internet use among undergraduate medical students of Delhi and derive a model for allocating new subjects among categories of internet users.

Material and methods: A cross-sectional study was conducted on 201 medical-undergraduate students in a medical college of Delhi from April 1st to May 31st, 2019. A semi-structured and pre-tested questionnaire was used to collect demographic information and factors affecting PIU. Dr. Kimberly Young's Internet Addiction Test (IAT) tool was used to assess PIU. Binary logistic regression has been applied to assess the correlates of PIU, and step-wise discriminant analysis (DA) has been applied to derive a model for allocation of new subjects among categories of internet users. Statistical Package for Social Sciences (Trial version 27.0; SPSS Inc., Chicago, IL) software was used for statistical analysis.

Result: Total $41.3 \%$ of the subjects had PIU. Univariate analysis shows that internet use for emotional support, watching adult content, and gambling were significantly associated with PIU; however, in binary logistic regression, chatting, emotional support and watching online adult content were significant risk factors for PIU. The discriminant model correctly classified $66.2 \%$ of respondents into average and problematic internet user groups.

Conclusion: We should create awareness among medical students regarding problematic internet use and its potential harms; this could be included in the foundation course of curriculum implementation support program (CISP) for MBBS students.
\end{abstract}

Keywords: Undergraduate medical, Internet, Discriminant analysis, Cross-sectional study, Problematic internet use

\section{Background}

The use of the internet has increased dramatically over the past two decades. Recent global internet statistics by global reach show over 803 million people have online access worldwide [1]. The benefits of the internet have been widely researched and include education and research, communication, health-related services, online monetary transactions, trade, buying goods, entertainment, etc. [2].

\footnotetext{
* Correspondence: drdeepakdhamnetiya@gmail.com

Department of Community Medicine, Dr Baba Saheb Ambedkar Medical College and Hospital, Sector-6 Rohini, New Delhi 110085, India
}

Researchers found that $73 \%$ of college students accessed the internet at least once a day and spent approximately 1.6 to $4.5 \mathrm{~h}$ a day online, preferably during the night [2-5]. Globally, due to population diversity, different instruments, cut-off scores used, and different sample characteristics, the prevalence of problematic internet use (PIU) vary from 7.3 to $51 \%$ [6-11]; whereas, In India, it ranges from 7.45 to $19.85 \%$ among undergraduate medical students $[2,3,12,13]$.

Globally, deteriorating effects of problematic internet use were studied by various researchers; they found that

(c) The Author(s). 2021 Open Access This article is licensed under a Creative Commons Attribution 4.0 International License, which permits use, sharing, adaptation, distribution and reproduction in any medium or format, as long as you give appropriate credit to the original author(s) and the source, provide a link to the Creative Commons licence, and indicate if changes were made. The images or other third party material in this article are included in the article's Creative Commons licence, unless indicated otherwise in a credit line to the material. If material is not included in the article's Creative Commons licence and your intended use is not permitted by statutory regulation or exceeds the permitted use, you will need to obtain permission directly from the copyright holder. To view a copy of this licence, visit http://creativecommons.org/licenses/by/4.0/ The Creative Commons Public Domain Dedication waiver (http://creativecommons.org/publicdomain/zero/1.0/) applies to the data made available in this article, unless otherwise stated in a credit line to the data. 
PIU was associated with a wide range of social and psychological problems, like academic failure, poor selfconfidence, poor psychological well-being, sleep deprivation, social withdrawal, poor diet, and cardiopulmonary complications [13-15]. The mean scores of the following domains: anxiety, depression, paranoid ideation, and obsessive-compulsive of Symptom Checklist90-revision (SCL-90-R), were lower in individuals without PIU vis-a-vis PIU [16, 17].

Researchers have suggested various socio-demographic, personal, and internet-related factors are associated with problematic internet use; male gender [4, 18], initial years during the study course, the influence of peers, always logged in status, online interaction with friends, chat, watching porn, online new friendships or relationships, online shopping, average daily time spent on internet and internet access modalities were some of the risk factors for problematic internet use $[2,3,18,19]$. However, correlates of problematic internet use among undergraduate medical students of North India remain underexplored. Moreover, no research studies have demonstrated a model predicting problematic internet use in a new subject. This study aims to assess the correlates of problematic internet use among undergraduate medical students of Delhi and derive a model for allocating new subjects among categories of internet users.

\section{Material and methods}

A cross-sectional study was conducted on 201 undergraduate students in a medical college of Delhi, India, from April 1st to May 31st, 2019. Inclusion criteria: All the undergraduate students studying in a medical college in Delhi and using the internet at least for the last six months were selected for the study. Exclusion criteria: those who do not give consent. Sample size calculation: A total of 300 students were enrolled in the medical college, 100 students in each batch. The sample size was calculated by taking prevalence of PIU as $50 \%$ at a level of $95 \%$ significance, $5 \%$ precision, and population size as 300 . $n=[\mathrm{Np}(1-\mathrm{p})] /\left[\left(\mathrm{d}^{2} / \mathrm{Z}^{2}{ }_{1-\alpha / 2}{ }^{*}(\mathrm{~N}-1)+\mathrm{p}^{*}(1-\mathrm{p})\right]\right.$.

Where, $n=$ sample size, $N=$ population size, $p=$ prevalence, $d=$ precision.

The required minimum sample size comes up to be 169; Considering a non-response rate of $10 \%$, the final minimum sample size was 188 . We have studied and analyzed data from 201 students.

\section{Questionnaire design and validation}

The pre-testing of semi-structured questionnaire was done on 20 undergraduate students. Some questions were modified after pre-testing. The language of some questions was improved for better clarity to the respondent. Reasons for internet use questions were changed from open-ended to 15 close-ended questions (as per responses received) with dichotomous responses "Yes" or "No", and one open-ended question were added for any other reason of internet use. This pre-tested questionnaire was used to collect information regarding age, gender, socio-economic status, place of residence, year of admission, ownership of gadget (computer, laptop, mobile, tablet), and questions related to internet use; preferred place of internet access (home, cybercafé, or others), for how long have you used the internet? On average, how much time per week do you spend on the internet? On average, how much money per month do you spend on the internet. Questions related to reasons for internet use; why do you use the internet (for communicating with friends and family, required for course work/ assignments, research on new developments/ in areas of interest, browsing, news updates, recreation or relaxation, meeting new people, chatting with others to share interests/ ideas or fantasies, time pass, emotional support, job search, gambling, adult-only content, games, and shopping, etc.). on an average, how much time per week do you spend on the internet sites like WhatsApp, online movies, online shopping, search tool (Google/ Bing, etc.), adult content site, email, torrent download, duration of internet use, money spent on the internet per month, Snapchat, Twitter, YouTube, Facebook, newsgroup, gaming sites, spiritual content, music/songs, and Instagram.

We have used Dr. Kimberly Young's internet addiction test (IAT) scale to assess PIU [20]. The IAT is a 20 -item that measures the severity of self-reported compulsive use of the internet. Each item is rated on a 6-point Likert scale ranging from 0 to 5; $0=$ Not Applicable, $1=$ Rarely, $2=$ Occasionally, $3=$ Frequently, $4=$ Often, $5=$ Always. The marking for this questionnaire ranges from 0 to 100; the higher the score range, the greater the level of addiction. Subjects with scores $<50$ were categorized as "average internet users," and those with scores $\geq 50$ were categorized as "problematic internet users. The Cronbach's $\alpha$ computed from the studies was 0.889 [95\% confidence interval (CI) 0.884-0.895]. The standard deviation of the alpha was low, at 0.049 [21]. In the present study, we have found high internal consistency, with an alpha coefficient of 0.889 (CI 0.867-0.911).

\section{Data collection}

We have stratified undergraduate students according to the year of admission and enrolled at least 50 students from each stratum. We have fixed the criteria that at least $60 \%$ of students should be present in each class. Out of the present students, $90 \%$ were chosen randomly using computer-generated random numbers by giving a serial number to the present students. Single attempts were made to collect data from each admission year student. A semi-structured and pre-tested questionnaire 
was distributed among randomly selected students, and they were asked to fill the questionnaire once. The researchers had explained the purpose and objectives of the study to the participants. Participants were informed that participation is voluntary and it will not affect their grades.

\section{Data management and statistical analysis}

Confidentiality of all the data was ensured by keeping the responses anonymous. Moreover, the collected data was stored under secure settings. Data was recorded in MS Excel, and the trial version of the statistical package for social sciences (version 27.0; SPSS Inc., Chicago, IL) software was used for statistical analysis. No missing data was encountered. Categorical data were described as frequencies and percentages. The unadjusted and adjusted odds ratio was calculated by applying binary logistic regression to assess the correlates of PIU. A $p$-value of less than 0.05 is considered significant for all analyses. Step-wise discriminant analysis (DA) has been applied to derive a model for allocating new subjects among categories of internet users.

The analysis creates a discriminant function, a linear combination of the weightings and scores on these variables. The maximum number of functions is either the number of predictors or the number of groups minus one, whichever of these two values is smaller [22]. The discriminant analysis involves determining a linear equation like a regression that will predict which group the case belongs to. The form of the equation or function is:

$Z_{j k}=\mathrm{a}+\mathrm{W}_{1} \mathrm{X}_{1 \mathrm{k}}+\mathrm{W}_{2} \mathrm{X}_{2 \mathrm{k}}+\ldots+\mathrm{W}_{\mathrm{n}} \mathrm{X}_{\mathrm{nk}}$.

Where:

$\mathrm{Z}_{\mathrm{jk}}=$ Discriminant $\mathrm{Z}$ score of discriminant function $\mathrm{j}$ for object $k$.

$\mathrm{a}=$ Intercept.

$\mathrm{W}_{\mathrm{i}}=$ Discriminant coefficient for the Independent variable $i$.

$\mathrm{X}_{\mathrm{ik}}=$ Independent variable $\mathrm{i}$ for object $\mathrm{k}$.

$n=$ number of predictor variables.

\section{Results}

In the present study, we have analyzed data of 201 subjects; the majority of the study subjects were $\geq 20$ years. Approximately $2 / 3 \mathrm{rd}$ of the subjects was males \& $96 \%$ followed the Hindu religion. Majority of the subjects (76.1\%) belongs to nuclear family and upper or uppermiddle SES (84.6\%). Approximately 3/4th of the study subjects $(74.1 \%)$ had permanent residence in Delhi, and more than half of the subjects $(55.7 \%)$ stayed in the hostel. $36.8 \%$ of the study subjects were in the third year, $36.3 \%$ in the second year, and $26.9 \%$ in the first year (Table 1).

Two third of the study subjects were started using the internet during their early adolescent period. Most of
Table 1 Distribution of study subjects according to sociodemographic characteristics $(N=201)$

\begin{tabular}{ll}
\hline Variable & Frequency (\%) \\
\hline Age & $59(29.4)$ \\
$\quad<20$ years & $142(70.6)$ \\
$\geq 20$ years & \\
Gender & $132(65.7)$ \\
Male & $69(34.3)$ \\
Female & \\
Religion & $193(96)$ \\
Hindu & $8(4)$ \\
Muslim or Sikh & \\
Type of family & $153(76.1)$ \\
Nuclear & $48(23.9)$ \\
Joint & \\
Socio-Economic Status & $170(84.6)$ \\
Upper \& Upper Middle & $31(15.4)$ \\
Lower \& Lower Middle & \\
Permanent residence & $149(74.1)$ \\
Delhi & $52(25.9)$ \\
Non-Delhi & \\
Hostel accommodation status & \\
Hosteller & $112(55.7)$ \\
Non-Hosteller & \\
Admission year & $(44.3)$ \\
\hline & \\
\hline &
\end{tabular}

the study subjects $(60.7 \%)$ used the internet for $6-10$ years, and only $11.4 \%$ of study subjects used the internet for more than ten years. Almost all the study subjects had smartphones (99\%), 51.7\% had laptops, 31.3\% had computers, and $24.4 \%$ had tablets. Only $15.4 \%$ of subjects had all the above electronic gadgets. Almost all the subjects (99\%) preferred smartphones for internet access. The majority of subjects access the internet daily, more than half $(51.7 \%)$ of the study subjects preferred night time to access the internet, and only $9 \%$ of subjects preferred morning time to access the internet. The majority of subjects $(72.1 \%)$ used the internet less than $5 \mathrm{~h}$ a day, and only $6 \%$ used the internet for more than $10 \mathrm{~h}$ a day. More than half $(50.7 \%)$ of the study subjects had spent less than INR 150 per month on the internet, and only $10 \%$ of study subjects had spent more than INR 500 per month on the internet. $60.7 \%$ of study subjects were permanently logged in, and $41.3 \%$ had PIU (Table 2).

Common reasons for internet usage found to be work or assignment, communication with friends, browsing, 
Table 2 Pattern of Internet use among study subjects $(N=201)$

\begin{tabular}{|c|c|c|}
\hline Variable & Frequency & Percentage \\
\hline \multicolumn{3}{|l|}{ Age at first internet use } \\
\hline $5-10$ years & 32 & 15.9 \\
\hline $11-15$ years & 133 & 66.2 \\
\hline $16-20$ years & 36 & 17.9 \\
\hline \multicolumn{3}{|l|}{ Duration of internet use } \\
\hline $1-5$ years & 56 & 27.9 \\
\hline $6-10$ years & 122 & 60.7 \\
\hline$>10$ years & 23 & 11.4 \\
\hline \multicolumn{3}{|c|}{ Ownership of electronic gadget with internet access ${ }^{*}$} \\
\hline Smartphone & 199 & 99 \\
\hline Laptop & 104 & 51.7 \\
\hline Computer & 63 & 31.3 \\
\hline Tablet & 49 & 24.4 \\
\hline All & 31 & 15.4 \\
\hline \multicolumn{3}{|c|}{ The most common mode of internet access } \\
\hline Smartphone & 199 & 99 \\
\hline Computer & 2 & 1 \\
\hline \multicolumn{3}{|l|}{ Internet use per week } \\
\hline 7 days & 192 & 95.5 \\
\hline $2-6$ days & 9 & 4.5 \\
\hline \multicolumn{3}{|c|}{ Preferred time to use internet } \\
\hline Day (6 am to 5 pm) & 18 & 9 \\
\hline Evening (5 pm-10 pm) & 79 & 39.3 \\
\hline Night (10 pm-5 am) & 104 & 51.7 \\
\hline \multicolumn{3}{|l|}{ Internet use per day } \\
\hline$\leq 5$ Hours & 145 & 72.1 \\
\hline $6-10$ Hours & 44 & 21.9 \\
\hline$>10$ Hours & 12 & 6.0 \\
\hline \multicolumn{3}{|c|}{ Money spent on the internet per month } \\
\hline INR 1-150 & 102 & 50.7 \\
\hline INR151-300 & 48 & 23.9 \\
\hline INR 301-500 & 31 & 15.4 \\
\hline INR $>500$ & 20 & 10 \\
\hline \multicolumn{3}{|l|}{ Log in status } \\
\hline Permanently login & 122 & 60.7 \\
\hline On and off & 79 & 39.3 \\
\hline \multicolumn{3}{|l|}{ Problematic internet use } \\
\hline Yes & 83 & 41.3 \\
\hline No & 118 & 58.3 \\
\hline
\end{tabular}

recreational or relaxation purposes, time pass, shopping, and news update, i.e., 99, 98.5, 98, 97.5, 96, 92.5, and 90\% respectively. About $21 \%$ of the subjects used the internet for gambling (Fig. 1). Univariate analysis shows that internet use for emotional support, watching adult content, and gambling were significantly associated with PIU; however, in binary logistic regression, chatting, emotional support and watching online adult content were found to be significant risk factors for PIU (Table 3).

In Table 4, The test of equality of group means has been performed to measure each independent variable's potential before the model is created. Each test displays the results of a one-way ANOVA for the independent variable using the grouping variable, i.e., Internet Users as the factor. If the $p$-value value is greater than 0.05 , the variable probably does not contribute to the model. Wilks' lambda is another measure of a variable's potential. Smaller values indicate the variable is better at discriminating between groups. We have found strong statistical evidence of significant differences between means of average internet users and problematic internet users for only seven variables naming email time (in min), shopping time (in min), YouTube time (in min), WhatsApp time (in min), movie time (in $\mathrm{min}$ ), download time (in $\mathrm{min}$ ) and educational use time (in min). In contrast, insignificant variables are not suitable to discriminate between average internet users and problematic internet users (Table 4).

The step-wise discriminant analysis method has been applied for selecting the "best" variables to use in the model. The step-wise method starts with a model that doesn't include any of the independent variables. At each step, the predictor with the largest $\mathrm{F}$ value to Enter a value that exceeds the entry criteria 3.84 is added to the model. At the last step, the variables left out of the analysis all have $\mathrm{F}$ to Enter values smaller than 3.84, so no more are added. So, the final selected variables in the model having $\mathrm{F}$ to enter value $>3.84$ are family income, email time, and WhatsApp time. The $\mathrm{F}$ value for a variable indicates its statistical significance in the discrimination between groups, i.e., it is a measure of the extent to which a variable makes a unique contribution to the prediction of a group membership.

The equation of the model while considering the variables selected by applying step-wise discriminant analysis is as follows:

$\mathrm{D}=\left(0.000^{*}\right.$ family income $)+\left(0.0076^{*}\right.$ email time $)+$ (0.001*WhatsApp time) -0.294 .

We can calculate the discriminant scores by putting the values of these three variables in the above discriminant equation, by comparing this discriminant score with the cut-off value (Fig. 2), we can predict the allocation of subjects in average internet users or problematic internet users' group.

Table 5 shows that $66.2 \%$ of respondents were correctly classified into average internet users and problematic internet users' groups. This model correctly predicts $92.4 \%$ of subjects with average internet use. 


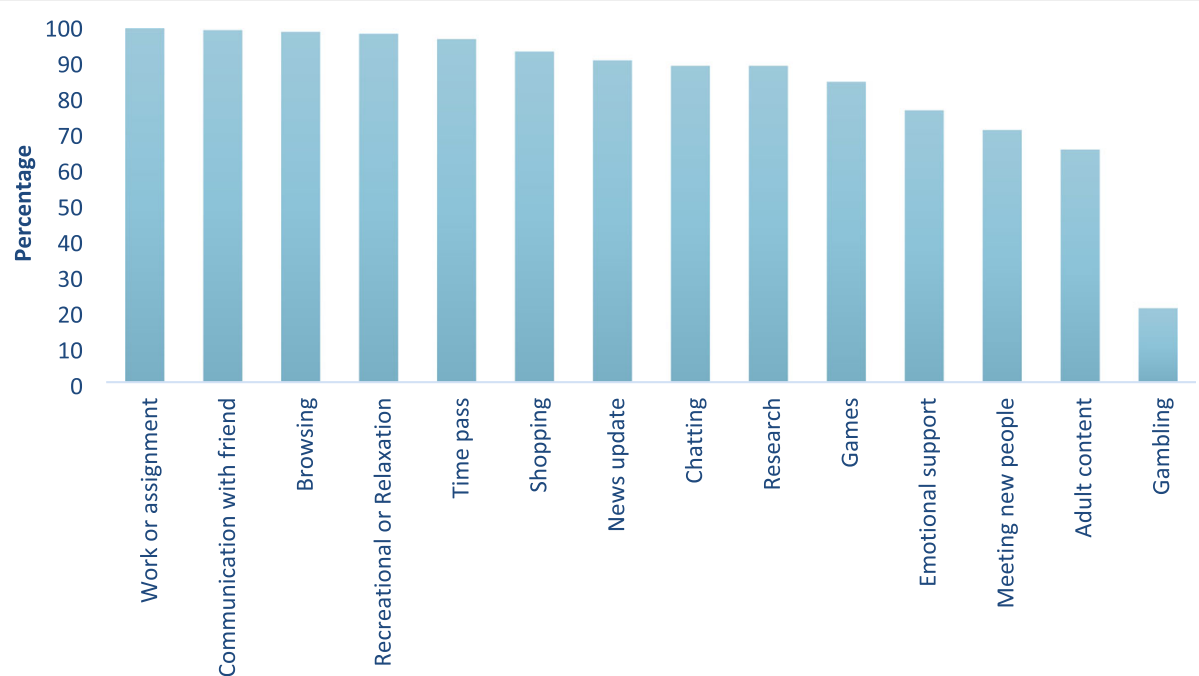

Fig. 1 Reasons of internet use among study subjects $(N=201)$

Figure 2 shows the discriminant scores of 201 subjects. The centroid value for average internet users is -0.278 , whereas for problematic internet users is 0.395 .

The general formula for the calculation of cut off value is given by

$$
Z_{c s}=\frac{N_{A} Z_{B}+N_{B} Z_{A}}{N_{A}+N_{B}}
$$

Where,

$\mathrm{Z}_{\mathrm{CS}}=$ Optimal cut-off value between groups $\mathrm{A}$ and $\mathrm{B}$. $\mathrm{N}_{\mathrm{A}}=$ number of observations in group $\mathrm{A}$.

Table 3 Association of selected risk factors with Problematic Internet Use $(N=201)$

\begin{tabular}{|c|c|c|c|c|}
\hline Variable & $\begin{array}{l}\text { Average Internet users } \\
\text { (118) }\end{array}$ & $\begin{array}{l}\text { Problematic Internet } \\
\text { users (83) }\end{array}$ & $\begin{array}{l}\text { Unadjusted OR } \\
(95 \% \mathrm{Cl})\end{array}$ & $\begin{array}{l}\text { Adjusted OR } \\
(95 \% \mathrm{Cl})\end{array}$ \\
\hline Gender (Male) & 76 & 56 & $1.15(0.63-2.08)$ & $0.68(0.32-1.46)$ \\
\hline Permanent residence (Delhi) & 93 & 59 & $1.51(0.79-2.89)$ & $0.49(0.21-1.10)$ \\
\hline Hostel accommodation status (Yes) & 67 & 45 & $0.90(0.51-1.59)$ & $0.63(0.31-1.31)$ \\
\hline Admission year (2016 \& 2017) & 85 & 62 & $1.15(0.61-2.17)$ & $1.352(0.65-2.80)$ \\
\hline Age at first internet use (> 10 Years) & 18 & 14 & $1.13(0.53-2.42)$ & $1.34(0.55-3.28)$ \\
\hline $\begin{array}{l}\text { Preferred time of day for internet access } \\
\text { (Evening or Night) }\end{array}$ & 105 & 78 & $1.931(0.66-5.64)$ & $2.62(0.78-8.86)$ \\
\hline Work or assignment (Yes) & 117 & 82 & $1.4(0.09-23.14)$ & $1.35(0.06-30.16)$ \\
\hline Communication with friend (Yes) & 117 & 81 & $2.90(0.26-32.39)$ & $1.31(0.08-20.98)$ \\
\hline Browsing (Yes) & 116 & 81 & $1.43(0.20-10.38)$ & $1.77(0.19-16.08)$ \\
\hline Recreational or Relaxation (Yes) & 115 & 81 & $0.95(0.16-5.79)$ & $0.24(0.02-3.38)$ \\
\hline Wasting time (Yes) & 112 & 81 & $0.46(0.09-2.34)$ & $1.14(0.17-7.50)$ \\
\hline Shopping (Yes) & 110 & 76 & $1.27(0.44-3.64)$ & $1.95(0.55-6.91)$ \\
\hline News update (Yes) & 107 & 74 & $1.18(0.47-3.00)$ & $0.96(0.31,2.99)$ \\
\hline Chatting (Yes) & 106 & 72 & $1.35(0.57-3.23)$ & $3.44(1.02-11.53) *$ \\
\hline Research (Yes) & 106 & 72 & $1.35(0.565-3.23)$ & $2.04(0.62-6.66)$ \\
\hline Games (Yes) & 98 & 71 & $0.83(0.38-1.80)$ & $0.77(0.30-1.99)$ \\
\hline Emotional support (Yes) & 81 & 72 & $0.33(0.16-0.70) *$ & $0.28(0.12-0.69) *$ \\
\hline Meeting new people (Yes) & 79 & 63 & $0.64(0.34-1.21)$ & $0.72(0.30-1.70)$ \\
\hline Adult content (Yes) & 69 & 62 & $0.48(0.26-0.88) *$ & $0.37(0.16-0.81) *$ \\
\hline Gambling (Yes) & 19 & 23 & $0.50(0.25-1.00) *$ & $0.65(0.29-1.46)$ \\
\hline
\end{tabular}

${ }^{*} p<0.05$ 
Table 4 Test of equality of group means of studied variables among categories of internet users

\begin{tabular}{llll}
\hline Variables & Wilks Lambda & F value & p-value \\
\hline Age & 0.995 & 1.096 & 0.296 \\
Family Income (INR) & 0.987 & 2.610 & 0.108 \\
Amount Spent on Internet (INR) & 0.997 & 0.570 & 0.451 \\
Email time (in min) & 0.972 & 5.651 & $0.018^{*}$ \\
Tool time (in min) & 0.985 & 3.042 & 0.083 \\
Newsgroup time (in min) & 0.988 & 2.432 & 0.120 \\
Game site time (in min) & 1.000 & 0.006 & 0.938 \\
Shopping time (in min) & 0.972 & 5.698 & $0.018^{*}$ \\
You Tube time (in min) & 0.977 & 4.607 & $0.033^{*}$ \\
Music time (in min) & 0.991 & 1.881 & 0.172 \\
Facebook time (in min) & 0.993 & 1.430 & 0.233 \\
WhatsApp time (in min) & 0.952 & 10.099 & $0.002^{*}$ \\
Twitter time (in min) & 0.989 & 2.304 & 0.131 \\
Instagram time (in min) & 0.995 & 1.001 & 0.318 \\
Snapchat time (in min) & 0.990 & 2.043 & 0.155 \\
Movie time (in min) & 0.971 & 5.883 & $0.016^{*}$ \\
Download time (in min) & 0.969 & 6.372 & $0.012^{*}$ \\
Educational use time (in min) & 0.972 & 5.733 & $0.018^{*}$ \\
Spiritual time (in min) & 0.992 & 1.664 & 0.199 \\
Adult site time (in min) & 0.986 & 2.854 & 0.093 \\
\hline * -Value 0.05 ( & &
\end{tabular}

* $p$-value $<0.05$
$\mathrm{N}_{\mathrm{B}}=$ number of observations in group $\mathrm{B}$.

$\mathrm{Z}_{\mathrm{A}}=$ Centroid for group $\mathrm{A}$.

$Z_{\mathrm{B}}=$ Centroid for group $\mathrm{B}$.

So, in this case, the cut-off score will be 0.118 . Hence the cut-off values above 0.118 are classified as problematic internet users, and below 0.118 are classified as average internet users.

\section{Discussion}

In our study, we have found $41.3 \%$ of the subject had PIU; this has been corroborated with a study conducted on medical students by Pramanik et al. [9]; whereas in some other studies, the PIU ranges from $5.8-30 \%[3,7$, $8,12,23-25]$; however, a study conducted by Sayyah et al. [11] found a high prevalence of PIU (51\%). The high magnitude of PIU in our study may be due to the demographic profile of study subjects as a majority (84.6\%) belongs to upper or upper-middle SES and increased penetration of internet in metro cities like Delhi. We have found no significant association between gender and PIU; similar results were found in a study conducted by other researchers [26, 27], whereas most of the studies showed that male gender was significantly associated with PIU [2, 3, 6, 7, 23, 25, 28, 29] However, previous studies found that females were significantly associated with PIU $[24,30]$. No significant association of gender with PIU in our study may be due to the fact that good accessibility to internet among male and female medical students. In this study, no association was seen between hostel accommodation and PIU; the same results were found by Salehi et al. [6] and Ghamari et al. [30]; whereas PIU was significantly higher in hostellers vis-a-vis non-hostellers in studies conducted by

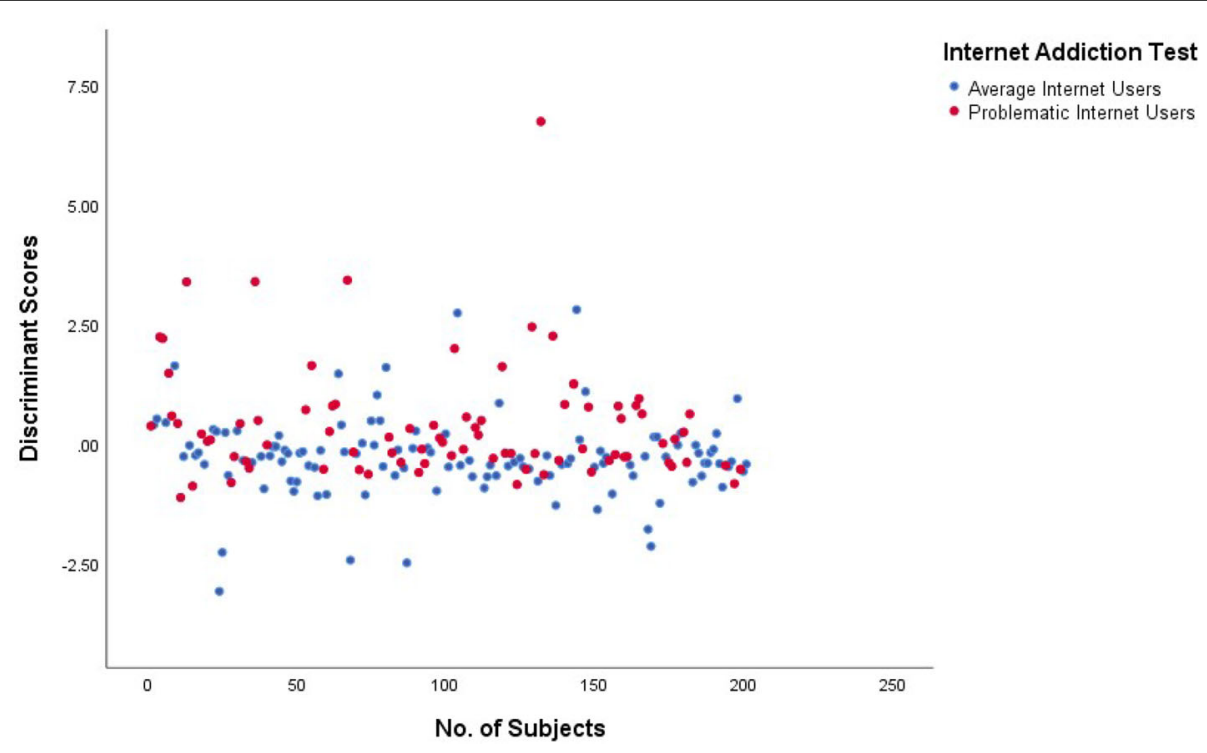

Fig. 2 Scatter plot of discriminant scores of each subject for the model 
Table 5 Classification result table of the proposed model by applying Step-wise discriminant analysis model $(N=201)$

\begin{tabular}{lclr}
\hline Original classification & \multicolumn{2}{c}{ Predicted Group Membership } & Total \\
\cline { 2 - 3 } & Average Internet Users (\%) & Problematic Internet Users (\%) & $118(58.7)$ \\
\hline Average Internet Users & $109(92.4)$ & $9(7.6)$ & $83(41.3)$ \\
Problematic Internet Users & $59(71.1)$ & $24(28.9)$ & \\
\hline
\end{tabular}

Chaudhari et al. [2] and Anand et al. [29]. We have found no association of PIU with the year of study, and the same result was found by Chaudhari et al. [2]. In contrast, Krishnamurthy et al. [12] and Asiri et al. [31] were found that students in first or second professional years had significant higher PIU as compare to thirdand fourth-year students; however, Sayyah et al. [11] found that PIU was significantly higher in senior students as compared to junior students. The reason of no association of PIU with the year of study in present study may be due to sharing of similar psychological and environment factors among medical students of all the professional years. We have found no significant association of PIU with age at first use of the internet. In contrast, some authors found age at first use of the internet was significantly lower in students with PIU $[2,28]$. We have found no association of PIU with a preferred time of internet use; this has been corroborated with a study conducted by Salehi et al. [6]; whereas Gedam et al. [3] found PIU was significantly higher in students whose preferred time of internet access was evening or night vis-a-vis morning or afternoon.

Our study shows that internet usage for emotional support, watching adult content, gambling and chatting was a statistically significant risk factor for PIU; this had been corroborated with studies conducted by several researchers $[2,7,12,23,32]$. We have found that internet usage for work or assignment, communication with a friend, browsing, recreational or relaxation, wasting time, shopping, news update, research, games, and meeting a new person on social media were not significantly associated with PIU, identical results were found in various studies conducted in India and other countries as well $[3,6,7,28]$. In contrast, Salehi et al. [6] found that communication with friends was significantly associated with PIU \& in a study conducted by Krishnamurthy et al. [12], internet use for work and making new friends on social media was significantly associated with PIU. A study conducted by Mazhari [7] found that the internet used for shopping was significantly associated with PIU. To the best of our knowledge, the present study is the first that discriminate a new subject in the average and problematic internet user groups. The model derived from step-wise DA suggests that family income, email time and WhatsApp time discriminate $66.2 \%$ of the subjects correctly into average and problematic internet user groups. WhatsApp is one of the commonest social networking applications used to share text massages, videos, photos, and work-related information which leads to its excessive use in everyday life [33]. Driving factor for the spread of WhatsApp use is its convenience; people may access massages and reply from anywhere anytime. Income is one of the factors that directly correlates with internet use. High income leads to more use of internet [34]. There is increase in the use of email for academic work, assignment and research related activity among medical students. These discriminators can be used to determine the PIU among undergraduate medical students.

Our study has several limitations; First, it's a singlecentre study, so multi-centre studies are warranted and explore the differences in areas, specialties, and grades. Second, being a cross-sectional study, we could not establish a cause-and-effect relationship; a longitudinal study would be more informative. Third, this study is subject to some recall bias. Fourth, the use of self-report for measuring time spent on a range of devices and activities by a person is likely to be biased.

\section{Conclusion}

Our study reported high PIU among undergraduate medical students. Internet usage for emotional support, watching online adult content, and chatting was significantly associated with PIU. We should create awareness among medical students regarding PIU and its potential harms; this could be included in the foundation course of the curriculum implementation support program (CISP) for MBBS students. The initiative should be taken to create ample opportunities for students to involve in extracurricular activities and interact with friends. There should be a provision of counsellors for emotional and mental support of medical students as they are overburden with studies and long posting schedules.

\footnotetext{
Abbreviations

Cl: Confidence Interval; CISP: Curriculum Implementation Support Program; DA: Discriminant Analysis; IAT: Internet Addiction Test; INR: Indian National Rupee; OR: Odds Ratio; PIU: Problematic Internet Use; SES: Socio-Economic Status
}

\section{Acknowledgments}

My sincere thanks to all the students who participated in this study.

\section{Authors' contributions}

DD and SVS prepared the study concept and design. DD and SVS wrote the main manuscript text. DD, SVS, and RPJ analyzed data and edited the draft. 
DD conducted the investigation and data collection. DD and SVS had full access to all data in the study and took responsibility for the integrity of the data and the accuracy of the data analysis. The author(s) read and approved the final manuscript.

\section{Funding}

Nil

\section{Availability of data and materials}

The study datasets are available from the corresponding author on reasonable request.

\section{Declarations}

\section{Ethics approval and consent to participate}

The informed written consent were obtained from all the participants. This study was conducted following the Declaration of Helsinki and was approved by the Institutional Ethical Committee of Dr. Baba Saheb Ambedkar Medical College and Hospital, Delhi (DBSAMC/10/EC/2019).

\section{Consent for publication}

Not applicable.

\section{Competing interests}

The authors declare that they have no competing interests.

\section{Received: 15 May 2021 Accepted: 8 October 2021}

\section{Published online: 15 October 2021}

\section{References}

1. Global reach (2019). Evolutions of online population. [Cited2019 March 11th]. Available from http://global-reach.biz/globstats/

2. Chaudhari B, Menon P, Saldanha D, Tewari A, Bhattacharya L. Internet addiction and its determinants among medical students. Ind Psychiatry J. 2015:24(2):15862

3. Gedam SR, Ghosh S, Modi L, Goyal A, Mansharamani H. Study of internet addiction: prevalence, pattern, and psychopathology among health professional undergraduates. Indian J Soc Psychiatry. 2017;33(4):305-11. https://doi.org/10.4103/ijsp.ijsp_70_16.

4. Scherer K. College life online: healthy and unhealthy internet use. J Coll Stud Dev. 1997:38:655-65.

5. Anderson K. Internet use among college students: an exploratory study. J Am Coll Heal. 2001;50(1):21-6. https://doi.org/10.1080/07448480109595707.

6. Salehi M, Khalili MN, Hojjat SK, Salehi M, Danesh A. Prevalence of internet addiction and associated factors among medical students from Mashhad, Iran in 2013. Iran Red Crescent Med J. 2014;16(5):e17256. https://doi.org/10. 5812/ircmj.17256.

7. Mazhari S. The prevalence of problematic internet use and the related factors in medical students, Kerman, Iran. Addict Health. 2012;4(3-4):87-94.

8. Zhang MWB, Lim RBC, Lee C, Ho RCM. Prevalence of internet addiction in medical students: a Meta-analysis. Acad Psychiatry. 2018;42(1):88-93. https:// doi.org/10.1007/s40596-017-0794-1.

9. Pramanik T, Sherpa MT, Shrestha R. Internet addiction in a group of medical students: a cross sectional study. Nepal Med Coll J. 2012;14(1):46-8.

10. Fineberg NA, Demetrovics Z, Stein DJ, loannidis K, Potenza MN, Grünblatt E, et al. Manifesto for a European research network into problematic usage of the internet. Eur Neuropsychopharmacol. 2018;28(11):1232-46. https://doi. org/10.1016/j.euroneuro.2018.08.004.

11. Sayyah M, Khanafereh S. Prevalence of internet addiction among medical students: a study from southwestern Iran. Cent Eur J Public Health. 2019; 27(4):326-9. https://doi.org/10.21101/cejph.a5171.

12. Krishnamurthy S, Chetlapalli SK. Internet addiction: prevalence and risk factors: a cross-sectional study among college students in Bengaluru, the Silicon Valley of India. Indian J Public Health. 2015:59(2):115-21. https://doi. org/10.4103/0019-557X.157531

13. Balhara YP, Gupta R, Atilola O, Knez R, Mohorović T, Gajdhar W, et al. Problematic internet use and its correlates among students from three medical schools across three countries. Acad Psychiatry. 2015;39(6):634-8. https://doi.org/10.1007/s40596-015-0379-9.

14. Christakis DA. Internet addiction: a 21st century epidemic? BMC Med. 2010; 8(1):61. https://doi.org/10.1186/1741-7015-8-61.
15. Çardak M. Psychological well-being and internet addiction among university students. Turk Online J Educ Tech. 2013;12.

16. Xiuqin H, Huimin Z, Mengchen L, Jinan W, Ying Z, Ran T. Mental health, personality, and parental rearing styles of adolescents with internet addiction disorder. Cyberpsychol Behav Soc Netw. 2010;13(4):401-6. https:// doi.org/10.1089/cyber.2009.0222.

17. Alavi SS, Alaghemandan H, Maracy MR, Jannatifard F, Eslami M, Ferdosi M. Impact of addiction to internet on a number of psychiatric symptoms in students of Isfahan universities, Iran, 2010. Int J Prev Med. 2012;3(2):122-7.

18. Paul AV, Ganapthi CK, Duraimurugan M, Abirami V, Reji E. Internet addiction and associated factors: a study among college students in South India. Innov J Med Health Sci. 2015;5(3):121-5.

19. Ceyhan AA. Predictors of problematic internet use on Turkish university students. CyberPsychol Behav. 2008;11(3):363-6. https://doi.org/10.1089/ cpb.2007.0112.

20. Young KS. Internet addiction test manual. Bradford: Center for Internet Addiction Recovery; 2007. https://doi.org/10.1002/9781118013991.

21. Frangos CC, Frangos CC, Sotiropoulos I. A meta-analysis of the reliability of Young's internet addiction test. In proceedings of the world congress on engineering 2012 Jul (Vol. 1, pp. 368-371). London, United Kingdom: World Congress on Engineering.

22. Ramayah T, Ahmad NH, Halim HA, Zainal SR, Lo MC. Discriminant analysis: an illustrated example. Afr J Bus Manag. 2010;4(9):1654-67.

23. Kim KM, Kim H, Choi JW, Kim SY, Kim JW. What types of internet services make adolescents addicted? Correlates of problematic internet use. Neuropsychiatr Dis Treat. 2020;16:1031-41. https://doi.org/10.2147/NDT.S247292

24. Haroon MZ, Zeb Z, Javed Z, Awan Z, Aftab Z, Talat W. Internet Addiction in Medical Students. J Ayub Med Coll Abbottabad. 2018;30(Suppl 1):S659-63.

25. Sharma A, Sahu R, Kasar PK, Sharma R. Internet addiction among professional courses students: a study from Central India. Int J Med Sci Public Health. 2014; 3(9):1069-73. https:/doi.org/10.5455/ijmsph.2014.180620142.

26. Ioannidis K, Chamberlain SR, Treder MS, Kiraly F, Leppink EW, Redden SA, et al. Problematic internet use (PIU): associations with the impulsive-compulsive spectrum. An application of machine learning in psychiatry. J Psychiatr Res 2016:83:94-102. https://doi.org/10.1016/j.jpsychires.2016.08.010.

27. Mamun MA, Hossain MS, Siddique AB, Sikder MT, Kuss DJ, Griffiths MD. Problematic internet use in Bangladeshi students: the role of sociodemographic factors, depression, anxiety, and stress. Asian J Psychiatr. 2019; 44:48-54. https://doi.org/10.1016/j.ajp.2019.07.005.

28. Ghamari F, Mohammadbeigi A, Mohammadsalehi N, Hashiani AA. Internet addiction and modeling its risk factors in medical students, Iran Indian. J Psychol Med. 2011;33(2):158-62. https://doi.org/10.4103/0253-7176.92068.

29. Anand N, Jain PA, Prabhu S, Thomas C, Bhat A, Prathyusha PV, et al. Internet use patterns, internet addiction, and psychological distress among engineering university students: a study from India. Indian J Psychol Med. 2018:40(5):458-67. https://doi.org/10.4103/IJPSYM.IJPSYM 13518.

30. Taha MH, Shehzad K, Alamro AS, Wadi M. Internet use and addiction among medical students in Qassim University, Saudi Arabia. Sultan Qaboos Univ Med J. 2019;19(2):e142-7. https://doi.org/10.18295/squmj.2019.19.02.010.

31. Asiri S, Fallahi F, Ghanbari A, Kazemnejad-Leili E. Internet addiction and its predictors in guilan medical sciences students, 2012. Nurs Midwifery Stud. 2013;2(2):234-9. https://doi.org/10.5812/nms.11626.

32. Mitchell KJ, Wells M. Problematic internet experiences: primary or secondary presenting problems in persons seeking mental health care? Soc Sci Med. 2007:65(6):1136-41. https://doi.org/10.1016/j.socscimed.2007.05.015.

33. Faye AD, Gawande S, Tadke R, Kirpekar VC, Bhave SH. WhatsApp addiction and borderline personality disorder: a new therapeutic challenge. Indian J Psychiatry. 2016 Apr;58(2):235-7. https://doi.org/10.4103/0019-5545.183790.

34. Martin SP, Robinson JP. The income digital divide: trends and predictions for levels of internet use. Soc Probl. 2007;54(1):1-22. https://doi.org/10.1525/ sp.2007.54.1.1.

\section{Publisher's Note}

Springer Nature remains neutral with regard to jurisdictional claims in published maps and institutional affiliations. 